\title{
BIOLOGICAL STUDY OF 3-(2-HYDROXY -3,4-BENZOPHENYL- 5- METHOXY)-5-ARYL -1-SUBSTITUTED PYRAZOLINES , PYRAZOLES AND 4-AROYL DERIVATIVES OF PYRAZOLINES
}

\author{
A.R.BIJWE, S.E.BHANDARKARa , *. \\ * Dept. of Chemistry, K.Z.S.Science college Bramhni Kalmeshwar (MS) India \\ E mail : archana.bijwe@ gmail.com \\ a Associate Professor, Dept.of Chemistry, G.V.I.S.H. ,Amaravati (MS) India \\ E mail : subodhvmv@gmail.com
}

\begin{abstract}
3-(2-hydroxy -3,4-benzophenyl 5-methoxy) 5-aryl -1-substituted pyrazolines , pyrazoles and 4-aroyl derivatives of pyrazolines were tested against pathogenic bacteria for their antimicrobial and antifungal activities by paper disc method. The organisms tested were Aspergillus niger, Escherichia coli, Proteus mirabilis and Pseudomonas aeruginasa.The solutions of these compounds were prepared in DMF solvent.The most of the compounds were found to be active against pathogens Aspergillus niger, Escherichia coli, Proteus mirabilis and Pseudomonas aeruginasa.
\end{abstract}

\section{Key Words: Antimicrobial activity, antifungal activities pyrazolines, pyrazoles. INTRODUCTION}

According to literature much work has been done on heterocyclic compounds for their antimicrobial activities on gram positive and gram negative bacteria.

Pyrazolines are known to posses antibacterial ${ }^{1-3}$ antifungal ${ }^{4-6}$, anti-inflammatory $^{7}$, insecticidal ${ }^{8}$, analgesic ${ }^{9}$, antipytretic ${ }^{10}$, and properties. Several pyrazolines are found important as pharmaceuticals. Pyrazoline derivatives acquire anti-implantation activity ${ }^{11}$ and cerebroprotective $^{12}$. pyrazoles exhibit a wide range of biological activities , viz. antipyretic ${ }^{13-14}$, antioxidant ${ }^{15}$, antiinvasive ${ }^{16}$, blood pressure lowering ${ }^{17}$, antidepressant ${ }^{18}$, antiinflammatory ${ }^{19}$, etc.

The present work deals with the study of antimicrobial activities of some newly synthesized pyrazoles and pyrazolines. These compounds were tested against Aspergillius niger, Escherichia coli, Proteus mirabilis and Pseudomonas aeruginasa. 


\section{EXPERIMENTAL}

Melting points were uncorrected. The structures of titled compounds were established on the basis of elemental analysis and spectral data.

The medium used throughout the experiment was HI-Media (Indian make) nutrient agar. For sterilization autoclave is used. The size of zones of inhibition were measured by antibiotic zone reader (Metzer Make).

\section{METHOD AND RESULTS}

The titled compounds were tested against pathogenic bacteria for their antimicrobial activities by paper disc method. The organisms tested were, Aspergillius niger, Escherichia coli, Proteus mirabilis and Pseudomonas aeruginasa. The solutions of these compounds were prepared in DMF solvent. The culture medium used was nutrient agar medium for antimicrobial study and the potato-dextrose-agar for antifungal study. After $24 \pm 2$ hours. of incubation at $37^{\circ} \mathrm{C} \pm 2^{\circ} \mathrm{C}$ the zones of inhibition were measured in $\mathrm{mm}$ and are recorded in Table No. 1 , 2 and 3

\section{TABLE -1}

Antimicrobial and antifungal activities of 3-(2-hydroxy-3,4benzophenyl-5methoxy)-5-aryl-1- substituted pyrazolines(4-6a, 4-6b,

$$
4-6 c, 4-6 d)
$$

\begin{tabular}{|l|l|c|l|l|c|}
\hline $\begin{array}{l}\text { Sr. } \\
\text { NO }\end{array}$ & Name of the compound & $\begin{array}{l}\text { E- } \\
\text { coli }\end{array}$ & $\begin{array}{l}\text { Pr. } \\
\text { mirabilis }\end{array}$ & $\begin{array}{l}\text { Ps. } \\
\text { aeruginosa }\end{array}$ & $\begin{array}{l}\text { A. } \\
\text { niger }\end{array}$ \\
\hline 1 & $\begin{array}{l}\text { 3-(2-Hydroxy-3,4-benzophenyl-5- } \\
\text { methoxy)-5-phenyl-1-carboxamido } \\
\text { pyrazoline (4a) }\end{array}$ & 7 & 8 & 18 & 16 \\
\hline 2 & $\begin{array}{l}\text { 3-(2-Hydroxy-3,4-benzophenyl-5- } \\
\text { methoxy)-5-phenyl-1- } \\
\text { thiocarboxamido pyrazoline (5a) }\end{array}$ & 16 & 11 & - & 7 \\
\hline 3 & 3-(2-Hydroxy-3,4-benzophenyl-5- & - & 11 & 8 & 7 \\
\hline
\end{tabular}




\begin{tabular}{|c|c|c|c|c|c|}
\hline & $\begin{array}{l}\text { methoxy)-5-phenyl-1- } \\
\text { isonicotinoylpyrazoline (6a) }\end{array}$ & & & & \\
\hline 4 & $\begin{array}{l}\text { 3-(2-Hydroxy-3,4-benzophenyl-5- } \\
\text { methoxy)-5-(4-methoxy phenyl)-1- } \\
\text { carboxamido pyrazoline (4b) }\end{array}$ & 14 & 17 & 10 & 11 \\
\hline 5 & $\begin{array}{l}\text { 3-(2-Hydroxy-3,4-benzophenyl-5- } \\
\text { methoxy)-5-(4-methoxy phenyl)-1- } \\
\text { thiocarboxamido pyrazoline (5b) }\end{array}$ & 8 & 9 & 7 & 7 \\
\hline 6 & $\begin{array}{l}\text { 3-(2-Hydroxy-3,4-benzophenyl-5- } \\
\text { methoxy)-5-(4-methoxy phenyl)-1- } \\
\text { isonicotinoyl pyrazoline (6b) }\end{array}$ & 10 & 16 & - & 8 \\
\hline 7 & $\begin{array}{l}\text { 3-(2-Hydroxy-3,4-benzophenyl)-5-(4- } \\
\text { hydroxy phenyl)-1-carboxamido } \\
\text { pyrazoline }(4 c)\end{array}$ & 16 & 16 & 8 & 7 \\
\hline 8 & $\begin{array}{l}\text { 3-(2-Hydroxy-3,4-benzophenyl-5- } \\
\text { methoxy)-5-(4-hydroxy phenyl)-1- } \\
\text { thiocarboxamido pyrazoline (5c) }\end{array}$ & 16 & - & - & 8 \\
\hline 9 & $\begin{array}{l}\text { 3-(2-Hydroxy-3,4-benzophenyl-5- } \\
\text { methoxy)-5-(4-hydroxy phenyl)-1- } \\
\text { isonicotinoyl pyrazoline (6c) }\end{array}$ & 16 & 14 & 12 & 14 \\
\hline 10 & $\begin{array}{l}\text { 3-(2-Hydroxy-3,4-benzophenyl-5- } \\
\text { methoxy)-5-(3,4-dimethoxy phenyl)-1- } \\
\text { carboxamido pyrazoline (4d) }\end{array}$ & 16 & 16 & 17 & 18 \\
\hline 11 & $\begin{array}{l}\text { 3-(2-Hydroxy-3,4-benzophenyl-5- } \\
\text { methoxy)-5-(3,4-dimethoxy phenyl)-1- } \\
\text { thiocarboxamido pyrazoline (5d) }\end{array}$ & 16 & - & 8 & - \\
\hline 12 & $\begin{array}{l}\text { 3-(2-Hydroxy-3,4-benzophenyl)-5- } \\
\text { (3,4-dimethoxyphenyl-5-methoxy)-1- } \\
\text { isonicotinoyl pyrazoline (6d) }\end{array}$ & - & 14 & 7 & - \\
\hline
\end{tabular}




\section{TABLE -2}

Antimicrobial and antifungal activities of 3-(2-hydroxy-3,4benzophenyl)-5-aryl-1- substituted pyrazoles (9-11a, 9-11b, 9-11c, 9-

11d)

\begin{tabular}{|c|c|c|c|c|c|}
\hline $\begin{array}{l}\text { Sr. } \\
\text { NO }\end{array}$ & Name of the compound & $\begin{array}{l}\text { E- } \\
\text { coli }\end{array}$ & $\begin{array}{l}\text { Pr. } \\
\text { mira } \\
\text { bilis }\end{array}$ & $\begin{array}{l}\text { Ps. } \\
\text { aerug } \\
\text { inosa }\end{array}$ & $\begin{array}{l}\text { A.nige } \\
\mathrm{r}\end{array}$ \\
\hline 1 & $\begin{array}{l}\text { 3-(2-Hydroxy-3,4-benzophenyl-5-methoxy)-5- } \\
\text { phenyl-1-carboxamido pyrazole (9a) }\end{array}$ & 8 & - & 8 & 6 \\
\hline 2 & $\begin{array}{l}\text { 3-(2-Hydroxy-3,4-benzophenyl-5-methoxy)-5- } \\
\text { phenyl-1-thiocarboxamido pyrazole (10a) }\end{array}$ & 9 & 7 & 7 & 8 \\
\hline 3 & $\begin{array}{l}\text { 3-(2-Hydroxy-3,4-benzophenyl)-5-phenyl-1- } \\
\text { isonicotinoyl pyrazole (11a) }\end{array}$ & 11 & - & 17 & 18 \\
\hline 4 & $\begin{array}{l}\text { 3-(2-Hydroxy-3,4-benzophenyl-5-methoxy)-5-(4- } \\
\text { methoxy phenyl)-1-carboxamido pyrazole (9b) }\end{array}$ & 18 & 12 & 16 & 14 \\
\hline 5 & $\begin{array}{l}\text { 3-(2-Hydroxy-3,4-benzophenyl)-5-(4-methoxy } \\
\text { phenyl)-1-thiocarboxamido pyrazole (10b) }\end{array}$ & - & 15 & - & 9 \\
\hline 6 & $\begin{array}{l}\text { 3-(2-Hydroxy-3,4-benzophenyl-5-methoxy)-5-(4- } \\
\text { methoxy phenyl)-1-isonicotinoyl pyrazole (11b) }\end{array}$ & 15 & 16 & - & 10 \\
\hline 7 & $\begin{array}{l}\text { 3-(2-Hydroxy-3,4-benzophenyl)-5-(4-nitro phenyl)- } \\
\text { 1-carboxamido pyrazole (9c) }\end{array}$ & 12 & 7 & 10 & 18 \\
\hline 8 & $\begin{array}{l}\text { 3-(2-Hydroxy-3,4-benzophenyl-5-methoxy)-5-(4- } \\
\text { nitro phenyl)-1-thiocarboxamido pyrazole (10c) }\end{array}$ & 12 & - & 14 & - \\
\hline 9 & $\begin{array}{l}\text { 3-(2-Hydroxy-3,4-benzophenyl-5-methoxy)-5-(4- } \\
\text { nitro phenyl)-1-isonicotinoyl pyrazole(11c) }\end{array}$ & 18 & 15 & 17 & 16 \\
\hline 10 & $\begin{array}{l}\text { 3-(2-Hydroxy-3,4-benzophenyl-5-methoxy)-5-(4- } \\
\text { chloro phenyl)-1-carboxamido pyrazole (9d) }\end{array}$ & - & 18 & - & 14 \\
\hline 11 & $\begin{array}{l}\text { 3-(2-Hydroxy-3,4-benzophenyl-5-methoxy)-5-(4- } \\
\text { chloro phenyl)-1-thiocarboxamido pyrazole (10d) }\end{array}$ & 17 & - & 18 & - \\
\hline 12 & $\begin{array}{l}\text { 3-(2-Hydroxy-3,4-benzophenyl)-5-(4-chloro } \\
\text { phenyl)-1-isonicotinoyl pyrazole (11d) }\end{array}$ & 18 & - & - & 8 \\
\hline
\end{tabular}




\section{TABLE -3}

Antimicrobial and antifungal activities of 3-(2-hydroxy-3,4benzophenyl-5-methoxy) -4-aroyl-5-aryl-1-substituted pyrazolines (13-15a, 13- 15b,13-15c,13-15d, 13-15e,13-15f,13-15g, 13-15h)

\begin{tabular}{|c|c|c|c|c|c|}
\hline Sr. NO & Name of the compound & $\begin{array}{l}\text { E- } \\
\text { coli }\end{array}$ & $\begin{array}{l}\text { Pr. } \\
\text { mirab } \\
\text { ilis }\end{array}$ & $\begin{array}{l}\text { Ps. } \\
\text { aeru } \\
\text { gino } \\
\text { sa }\end{array}$ & $\begin{array}{l}\text { A.nige } \\
r\end{array}$ \\
\hline 1 & $\begin{array}{l}\text { 3-(2-Hydroxy-3,4-benzophenyl-5- } \\
\text { methoxy)-4-benzoyl-5- phenyl -1- } \\
\text { carboxamido pyrazoline (13a) }\end{array}$ & 11 & 10 & 18 & 16 \\
\hline 2 & $\begin{array}{l}\text { 3-(2-Hydroxy-3,4-benzophenyl-5- } \\
\text { methoxy)-4-benzoyl-5- phenyl -1- } \\
\text { thiocarboxamido pyrazoline (14a) }\end{array}$ & 15 & - & - & 12 \\
\hline 3 & $\begin{array}{l}\text { 3-(2-Hydroxy-3,4-benzophenyl-5- } \\
\text { methoxy)-4-benzoyl-5- phenyl -1- } \\
\text { isonicotinoyl pyrazoline (15a) }\end{array}$ & - & 12 & 15 & 16 \\
\hline 4 & $\begin{array}{l}\text { 3-(2-Hydroxy-3,4-benzophenyl-5- } \\
\text { methoxy)-4-benzoyl-5-(4-methoxy } \\
\text { phenyl)-1-carboxamido pyrazoline (13b) }\end{array}$ & 8 & - & - & 12 \\
\hline 5 & $\begin{array}{l}\text { 3-(2-Hydroxy-3,4-benzopheny-5-methoxy } \\
\text { 1)-4-benzoyl-5-(4-methoxy phenyl)-1- } \\
\text { thiocarboxamido pyrazoline (14b) }\end{array}$ & 17 & 11 & 8 & - \\
\hline 6 & $\begin{array}{l}\text { 3-(2-Hydroxy-3,4-benzophenyl-5- } \\
\text { methoxy)-4-benzoyl-5-(4-methoxy } \\
\text { phenyl)-1- isonicotinoyl pyrazoline (15b) }\end{array}$ & - & - & 12 & 8 \\
\hline 7 & $\begin{array}{l}\text { 3-(2-Hydroxy-3,4-benzophenyl-5- } \\
\text { methoxy)-4-benzoyl-5-(4-hydroxy phenyl)- } \\
\text { 1-carboxamido pyrazoline (13c) }\end{array}$ & 10 & 10 & 16 & - \\
\hline 8 & $\begin{array}{l}\text { 3-(2-Hydroxy-3,4-benzophenyl-5- } \\
\text { methoxy)-4-benzoyl-5-(4-hydroxy phenyl)- } \\
\text { 1-thiocarboxamido pyrazoline(14c) }\end{array}$ & 10 & - & - & 8 \\
\hline 9 & $\begin{array}{l}\text { 3-(2-Hydroxy-3,4-benzophenyl-5- } \\
\text { methoxy)-4-benzoyl-5-(4-hydroxy phenyl)- }\end{array}$ & 12 & 14 & 14 & - \\
\hline
\end{tabular}




\begin{tabular}{|c|c|c|c|c|c|}
\hline & 1-isonicotinoyl pyrazoline (15c) & & & & \\
\hline 10 & $\begin{array}{l}\text { 3-(2-Hydroxy-3,4-benzophenyl-5- } \\
\text { methoxy)-4-benzoyl-5-(3,4-dimethoxy } \\
\text { phenyl)-1-carboxamido pyrazoline (13d) }\end{array}$ & - & - & 14 & 7 \\
\hline 11 & $\begin{array}{l}\text { 3-(2-Hydroxy-3,4-benzophenyl-5- } \\
\text { methoxy)-4-benzoyl-5-(3,4-dimethoxy } \\
\text { phenyl)-1-thiocarboxamido } \\
\text { pyrazoline(14d) }\end{array}$ & 15 & - & 10 & 7 \\
\hline 12 & $\begin{array}{l}\text { 3-(2-Hydroxy-3,4-benzophenyl-5- } \\
\text { methoxy)-4-benzoyl-5-(3,4-dimethoxy } \\
\text { phenyl)-1- isonicotinoyl pyrazoline (15d) }\end{array}$ & - & 16 & 13 & 8 \\
\hline 13 & $\begin{array}{l}\text { 3-(2-Hydroxy-3,4-benzophenyl-5- } \\
\text { methoxy)-4-(4-methoxy benzoyl)-5- } \\
\text { phenyl-1-carboxamido pyrazoline (13e) }\end{array}$ & 9 & 11 & 14 & 8 \\
\hline 14 & $\begin{array}{l}\text { 3-(2-Hydroxy-3,4-benzophenyl-5- } \\
\text { methoxy)-4-(4-methoxy benzoyl)-5- } \\
\text { phenyl-1-thiocarboxamido pyrazoline } \\
(14 \mathrm{e})\end{array}$ & - & 11 & 14 & 8 \\
\hline 15 & $\begin{array}{l}\text { 3-(2-Hydroxy-3,4-benzophenyl-5- } \\
\text { methoxy)-4-(4-methoxy benzoyl)-5- } \\
\text { phenyl-1-isonicotinoyl pyrazoline (15e) }\end{array}$ & - & 9 & 16 & 14 \\
\hline 16 & $\begin{array}{l}\text { 3-(2-Hydroxy-3,4-benzophenyl-5- } \\
\text { methoxy)-4-(4-methoxy benzoyl)-5-(4- } \\
\text { methoxy phenyl)-1-carboxamido } \\
\text { pyrazoline (13f) }\end{array}$ & - & 8 & 16 & 13 \\
\hline 17 & $\begin{array}{l}\text { 3-(2-Hydroxy-3,4-benzophenyl-5- } \\
\text { methoxy)-4-(4-methoxy benzoyl)-5-(4- } \\
\text { methoxy phenyl)-1-thiocarboxamido } \\
\text { pyrazoline (14f) }\end{array}$ & - & 11 & - & 12 \\
\hline 18 & $\begin{array}{l}\text { 3-(2-Hydroxy-3,4-benzophenyl-5- } \\
\text { methoxy)-4-(4-methoxy benzoyl)-5-(4- } \\
\text { methoxy phenyl)-1-isonicotinoyl } \\
\text { pyrazoline (15f) }\end{array}$ & 15 & 8 & - & 8 \\
\hline 19 & $\begin{array}{l}\text { 3-(2-Hydroxy-3,4-benzophenyl-5- } \\
\text { methoxy)-4-(4-methoxy benzoyl)-5-(4- } \\
\text { hydroxy phenyl)-1-carboxamido }\end{array}$ & 13 & 7 & - & 8 \\
\hline
\end{tabular}




\begin{tabular}{|c|c|c|c|c|c|}
\hline & pyrazoline (13g) & & & & \\
\hline 20 & $\begin{array}{l}\text { 3-(2-Hydroxy-3,4-benzophenyl-5- } \\
\text { methoxy)-4-(4-methoxy benzoyl)-5-(4- } \\
\text { hydroxy phenyl)-1-thiocarboxamido } \\
\text { pyrazoline (14g) }\end{array}$ & 11 & 13 & - & 12 \\
\hline 21 & $\begin{array}{l}\text { 3-(2-Hydroxy-3,4-benzophenyl-5- } \\
\text { methoxy)-4-(4-methoxy benzoyl)-5-(4- } \\
\text { hydroxy phenyl)-1-isonicotinoyl pyrazoline } \\
(15 \mathrm{~g})\end{array}$ & - & 8 & 16 & 14 \\
\hline 22 & $\begin{array}{l}\text { 3-(2-Hydroxy-3,4-benzophenyl-5- } \\
\text { methoxy)-4-(4-methoxy benzoyl)-5-(3,4- } \\
\text { dimethoxy phenyl)-1-carboxamido } \\
\text { pyrazoline (13h) }\end{array}$ & 16 & - & 17 & 10 \\
\hline 23 & $\begin{array}{l}\text { 3-(2-Hydroxy-3,4-benzophenyl-5- } \\
\text { methoxy)-4-(4-methoxy benzoyl) -5-(3,4- } \\
\text { dimethoxy phenyl)-1-thiocarboxamido } \\
\text { pyrazoline }(14 \mathrm{~h})\end{array}$ & 11 & 14 & - & 10 \\
\hline 24 & $\begin{array}{l}\text { 3-(2-Hydroxy-3,4-benzophenyl-5- } \\
\text { methoxy)-4-(4-methoxy benzoyl) -5-(3,4- } \\
\text { dimethoxy phenyl)-1- isonicotinoyl } \\
\text { pyrazoline (15h) }\end{array}$ & 15 & - & 14 & 12 \\
\hline
\end{tabular}

Strongly active, range 15-18 Weakly active, range $7-10 \mathrm{~mm}$ Moderately active, range 11-14mm Inactive, -

\section{CONCLUSION}

Thus from above results it was observed that most of the heterocyclic compounds are found to be active against Escherichia coli, Proteus mirabilis, Pseudomonas aeruginosa and Aspergillius niger. So these compounds can be easily be used for the treatment of diseases caused by test pathogens, only when they do not have toxic and other side effects.

\section{ACKNOWLEDGEMENT}


The authors are thankful to the Director of LIT, Nagpur University Nagpur for providing facilities for testing the compounds against pathogenic bacterias and for their kind cooperation.

\section{REFERENCES}

Pandeya, S. N.; and Sriram, D. (1998). Acta Pharm. Turc. 40, 33.

Sarangapani, M.; and Reddy, V. M. (1994), Indian J. Pharm. Sci. 56, 174.

Varma, R. S.; Nobles, W. L. J. Pharm. Sci.64, (1975), 1.

Pandeya, S. N.; Sriram, D.; Nath, G.; and De Clercq, (1999). E. Indian J. Pharm. Sci. 61, 358.

Pandeya, S. N.; Sriram, D.; Nath, and G.; De Clercq, (1999). E. Sci. Pharm. 67, 103.

Pandeya, S.N.; Sriram, D.; Nath, and G.; De Clercq, ,(1999), E.; Pharm. Acta Helv. , 74,11.

Udupi R.H., Kushnoor AS and Bhat AR, (1998). Indian J Heterocycl Chem , 8, ,63.

Hes R V Wellinga K and Gregoy AC, (1975). JAgric Food Chem, 26, 1057 .

M.A. Metwally, M.Y. Yusuf, A.M. Tsmaiel and F.A.Amer, (1985) . J. Indian Chem., Soc.,62,54.

V.G.Vornin, Z.I. Sharmova, S. Ya. Shachilova, L.D. Kulikova and A.S. Zaks. (1985). Khim, Farm. Zh., 19, 1208; Chem. Abstr., 104, $61668 \mathrm{~m}$.

Batulin Y. U. M. Chem. Abstr., 70, (1969), 2236a

G. E. H. Elgemeie, A. M. E. Attia, D. S. Farag, and S. M. Sherif, (1994). J. Chem. Soc. Perkin Trans., 1, 1285. 
.Behr L C, Fusco R and Jarboe C H, (1967). The Chemistry of Heterocyclic Compounds, Pyrazoles, Pyrazolines ,pyrazolidines, Indazoles, and Coindensed Rings, edited by A Weissberger, Interscience Publishers, New York, 1.

Wiley R H, and Wiley P, (1964). Pyrazolones pyrazolidones and Derivatives, John Wiley and Sons, New York, 102.

Parmar V. S. , Kumar A, Prasad AK,Singh SK, Kumar N, Mukherjee s,Raj HG, Goel S,Errington W and Puar S, (1999). Bioorg Med Chem,17, 1425.

Parmar V S, bracke M Et al, Philippe J, Wengel J, Jain S C, Olsen C E, Bisht K S, Sharma N K, Courtens A, Sharma S K, Vennekens K, Marck V V, Singh S K, Kumar N, Kumar A, Malhotra S, Kumar R, Rajwanshi V K, Jain R, and Mareel M M, (1997). Bioorg Med Chem 5, 1609 .

Rosiere C E and Grossmann M I, (1951). Science, 113,651.

Bailey D M, Hansen P E, Hlavac A G, Baizman E R, Pearl J, Defelice A F, and Feigenson M E , (1985). J Med Chem, 28, 256.

Rainer G, Krueger U, and Klemm K, Arzneim Forsh, 31, (1981).

649;Chem Abstr, 95, (1981), 90723. 